\title{
The process of distribution Computer modeling of hazardous substances in the atmosphere taking into account the terrain relief
}

\author{
Daler Sharipov ${ }^{1 *}$, Zafar Abdullaev², and Otabek Khafizov² \\ ${ }^{1}$ Tashkent University of Information Technology named after Muhammad al-Khwarizmi, Tashkent, \\ Uzbekistan \\ ${ }^{2}$ Tashkent Institute of Irrigation and Agricultural Mechanization Engineers, Tashkent, Uzbekistan
}

\begin{abstract}
The paper considers a mathematical model for research, forecasting, and management decisions on the process of spreading harmful aerosol substances in the atmosphere. When deriving a mathematical model of the object, the main weather and climatic factors affecting the process of transport and diffusion of harmful substances and the terrain of the region under consideration are taken into account. Changes in wind direction and speed are calculated using the NavierStokes equation of stream function and vortex velocity variables. The proposed software was implemented as a software tool in the Borland $\mathrm{C}++$ Builder environment to carry out computational experiments. The results of the performed numerical calculations are shown in the form of graphical objects.
\end{abstract}

\section{Introduction}

Since the ecology of the atmosphere is one of the most important indicators of the state of the environment, these circumstances make it necessary to forecast the concentration of impurities in the surface layer of the atmosphere for different time frames. In particular, of practical interest are short-term forecasts related to the envisaged maximum permissible concentration of harmful impurities in the design of new enterprises.

Pollution of the surface layer of the atmosphere and the underlying surface, including the transport and diffusion of harmful substances, as well as their deposition and concentration, is a very complex process influenced by many factors, including geographic and weather, and climatic conditions typical for a particular region under consideration. Moreover, it is important to consider the fact that meteorological conditions change during the day and depending on the terrain.

The problem of mathematical modeling of the process of transport and diffusion of harmful substances in the boundary layer of the atmosphere is actively solved by many scientists in Uzbekistan and abroad. On the topic of this problem, such outstanding researchers as G.I.Marchuk, M.I.Lunev, M.E.Berlyand, V.V.Penenko, V.M.Belolipetskaya, F.B.Abutaliev, S.K.Karimberdieva and other, entire scientific schools were created that are

\footnotetext{
*Corresponding author: abdullaev.zafar.70@mail.ru
} 
effectively working today.

In particular, work [1] is devoted to developing a mathematical model of the dynamics and kinetics of the process of transport and diffusion of gaseous and aerosol impurities in the atmosphere.

The work [2] presents the developed mathematical support for the process of placing fire hazardous objects and their optimization, taking into account the terrain and spatial form.

The authors of [3] carried out a study based on the developed regional models of the diffusion process of substances described by the equation of molecular thermal conductivity in the active layer of the soil, taking into account the heat balance of the underlying surface. The authors investigated the environmental problems associated with the distribution of pollutants from known sources and the determination of the likely location of the source.

The process of transport and diffusion of harmful substances in the atmosphere, considering various weather and climatic factors and external disturbances, was considered in [4].

Work [5] is devoted to the process of dispersion and diffusion of chemically active primary pollutants emitted from elevated linear sources into a stable boundary layer of the atmosphere with a generalized wind speed and a quadratic function of vertical height. The authors proposed an exact solution to the problem using the Laplace transform, taking into account the ongoing chemical reaction, a change in the state of aggregation of particles, and their deposition on the underlying surface.

Important results on numerical modeling of the turbulence processes and diffusion of impurities in the surface layer of the atmosphere are contained in the works of D.L. Leichtman [6].

Significant achievements in the field of mathematical modeling of atmospheric processes are contained in the works of A.E. Aloyan [7]. In particular, the author considers a mathematical model of the transport of a multicomponent impurity, taking into account photochemical transformation and the formation of aerosols in the troposphere.

The main approaches to solving the problem of identifying aerosols of various origins and the research results are presented in the work of V.K. Danchenko and T.C. Ivleva [8], [9].

In the works of G.I. Marchuk and his followers [10], developed a full-fledged methodology of mathematical modeling, investigated its fundamental issues, and proposed original constructive approaches to studying the circulation of the atmosphere and ocean, as well as to solving problems of weather forecasting, climate theory and environmental protection using mathematical models.

To date, the influence of wind speed on the spread of harmful substances has been considered in the works of Professor F.N. Yasinsky [11]. Accounting for the stream function and vortex of velocities is considered in the works of R.V. Mayer and his students [12].

\section{Materials and Methods}

We consider a two-dimensional equation for the transport and diffusion of harmful substances in the atmosphere for mathematical modeling of the process of transport and diffusion of harmful substances in the atmosphere, taking into account the orography of the earth's surface as the first boundary-value problem $[13,14]$ : 


$$
\begin{aligned}
& \frac{\partial \varphi}{\partial t}+u \frac{\partial \varphi H}{\partial x}+\left(w-w_{g}\right) \frac{\partial \varphi H}{\partial z}+\sigma \varphi H= \\
& =\mu \frac{\partial^{2} \varphi H}{\partial x^{2}}+\frac{\partial}{\partial z}\left(\lambda H \frac{\partial \varphi}{\partial z}\right)+\delta_{i, k} f,
\end{aligned}
$$

with the following initial and boundary conditions

$$
\begin{aligned}
& \left.\varphi(x, y, z, t)\right|_{t=0}=\varphi_{0}(x, y, z), \\
& \left.\alpha_{1}\left(\varphi-\varphi_{o k}\right)\right|_{x=0}+\left.\beta_{1} \frac{\partial \varphi}{\partial x}\right|_{x=0}=0, \\
& \left.\alpha_{2}\left(\varphi-\varphi_{o k}\right)\right|_{x=L_{1}}+\left.\beta_{2} \frac{\partial \varphi}{\partial x}\right|_{x=L_{1}}=0, \\
& \lambda \frac{\partial \varphi}{\partial z}-H \beta \varphi=-H f_{0} \quad \text { at } z=0, \\
& \left.\alpha_{3}\left(\varphi-\varphi_{o k}\right)\right|_{z=L_{2}}+\left.\beta_{3} \frac{\partial \varphi}{\partial x}\right|_{z=L_{2}}=0,
\end{aligned}
$$

These $\varphi_{0}, \varphi_{o k}, \varphi$ are initial concentration, concentration at the boundaries of the area, concentration of the area under consideration in the atmosphere; $u, w$ is wind speed in two directions is determined from the Navier-Stokes equation; $w_{g}$ is particle settling rate; $\sigma$ is absorption coefficient; $\beta$ is underlying surface coefficient; $\mu, \lambda$ are diffusion and turbulence coefficients; $\delta_{i, k}$ is Dirac function; $f, f_{0}$ are sources of emission of harmful substances from industrial sites and the settling surface of the earth. Options $\alpha_{1}, \alpha_{2}, \alpha_{3}, \beta_{1}, \beta_{2}, \beta_{3}$ in equations (3)-(6) are set depending on the formulation of the problem and can be 0 or 1 . $H$ is parameter for determining the terrain, sought using the relation, L1, L2 is the length of the problem solution area, respectively in $\mathrm{x}$ and $\mathrm{z}$ [15], [16].

$$
H=\left\{\begin{array}{l}
0-\text { if the layer is underground } \\
1-\text { if the layer is in the atmosphere; } \\
\left(\eta-z_{K-0,5}\right) / \Delta z-\text { if the layer is below the orographic surface. }
\end{array}\right.
$$

Here $\eta$ is height of elevation below a plane parallel to sea level $\Delta z=z_{k+0,5}-z_{k-0,5}$. For each layer of the model, a multiplier $H(0 \leq H \leq 1)$, is introduced that determines the degree of blocking of the air flow (Fig.1). 


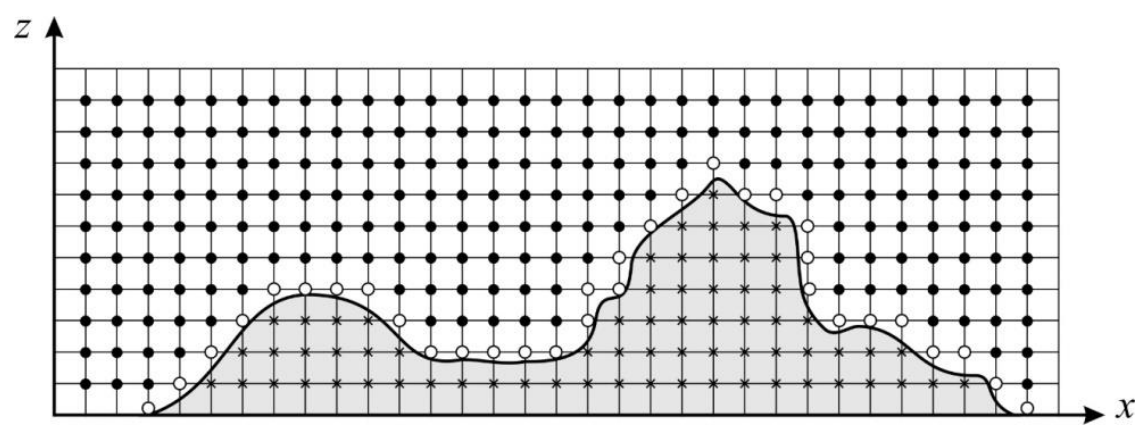

Fig.1. Terrain relief along the aerosol emission transport area.

$$
\text { ○. } H=\left(\eta-z_{K-0,5}\right) / \Delta z, \times-H=0, \bullet-H=1 \text {. }
$$

To determine the wind speed, we use the Navier - Stokes equations:

$$
\left\{\begin{array}{l}
\frac{\partial u}{\partial t}+u \frac{\partial u}{\partial x}+w \frac{\partial u}{\partial z}=\frac{1}{\rho} \frac{\partial p}{\partial x}+v\left(\frac{\partial^{2} u}{\partial x^{2}}+\frac{\partial^{2} u}{\partial z^{2}}\right) \\
\frac{\partial w}{\partial t}+u \frac{\partial w}{\partial x}+w \frac{\partial w}{\partial z}=\frac{1}{\rho} \frac{\partial p}{\partial x}+v\left(\frac{\partial^{2} w}{\partial x^{2}}+\frac{\partial^{2} w}{\partial z^{2}}\right) \\
\frac{\partial u}{\partial x}+\frac{\partial w}{\partial z}=0
\end{array}\right.
$$

Let's turn to the system of Navier-Stokes equations in variables of stream function and vortex velocity [16], [17]. We introduce a stream function such that:

$$
u=\frac{\partial \psi}{\partial z}, \quad w=\frac{\partial \psi}{\partial x} .
$$

The motion is two-dimensional, and the vorticity vector is perpendicular to the plane of the figure and is equal to:

$$
\omega=\frac{\partial u}{\partial z}-\frac{\partial w}{\partial x}=\frac{\partial^{2} \psi}{\partial x^{2}}+\frac{\partial^{2} \psi}{\partial z^{2}} .
$$

Suppose there is no pressure gradient. If the first equation from the Navier - Stokes system is differentiated concerning $\mathrm{z}$, the second concerning $\mathrm{x}$, then we obtain [18-20]:

$$
\frac{\partial \omega}{\partial t}+u \frac{\partial \omega}{\partial x}+w \frac{\partial \omega}{\partial z}=v\left(\frac{\partial^{2} \omega}{\partial x^{2}}+\frac{\partial^{2} u}{\partial z^{2}}\right)
$$

The last three equations make up the "stream function - vortex of velocity" system. The boundary condition for equations (7) - (10) are specified as in Fig. 2. 


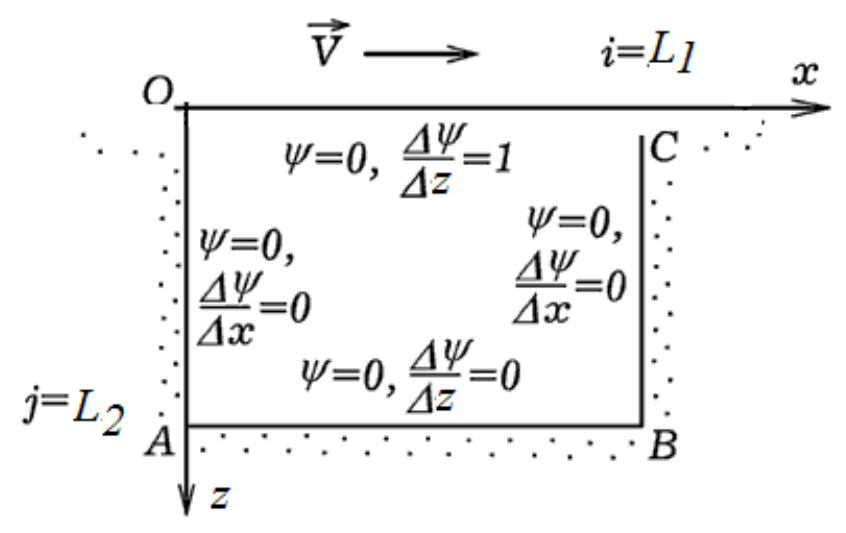

Fig.2. Boundary conditions and air movement in a closed space with an open surface.

\section{Results and Discussion}

From the statement of the problem (1) - (6) and equations (7) - (10), it follows that it is difficult to obtain an analytical solution. Therefore, for the numerical integration of the problem, a numerical algorithm was developed based on replacing differential operators with finite-difference operators [14-15].

The conducted studies of the process of the spread of harmful substances in the atmosphere showed that depending on the speed of the air flow and its direction, the dynamics of changes in the concentration of impurities in the atmosphere significantly fluctuate in time due to the stream function - the vortex of speed on the earth's surface.

Computational experiment and analysis of numerical calculations. Based on the presented mathematical model and numerical algorithm, a software tool was developed in the Borland $\mathrm{C}++$ Builder language. This program allows you to calculate the twodimensional movement of an air flow in a closed space with an open surface at different speeds and wind directions.

In fig. 3 shows the resulting distribution of the stream function. Borders of different shades correspond to streamlines. In principle, it is easy to construct streamlines by running marker particles inside the cavity. 


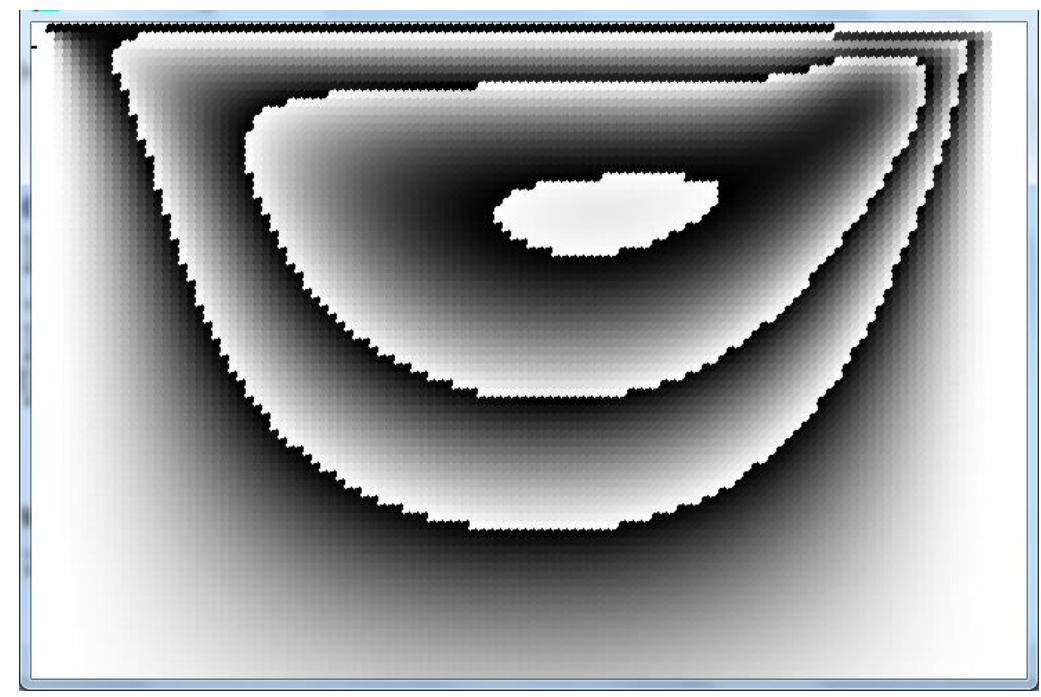

Fig.3. Borders of different shades of gray correspond to streamlines.

The software tool has a modular structure. The main module is designed to prepare and enter the main parameters of the process (air mass velocity, diffusion coefficient, absorption coefficient of harmful substances in the atmosphere, source power, computer calculation time, integration step in time and space variables, type of ingredient, coordinates of the emitter of harmful substances).

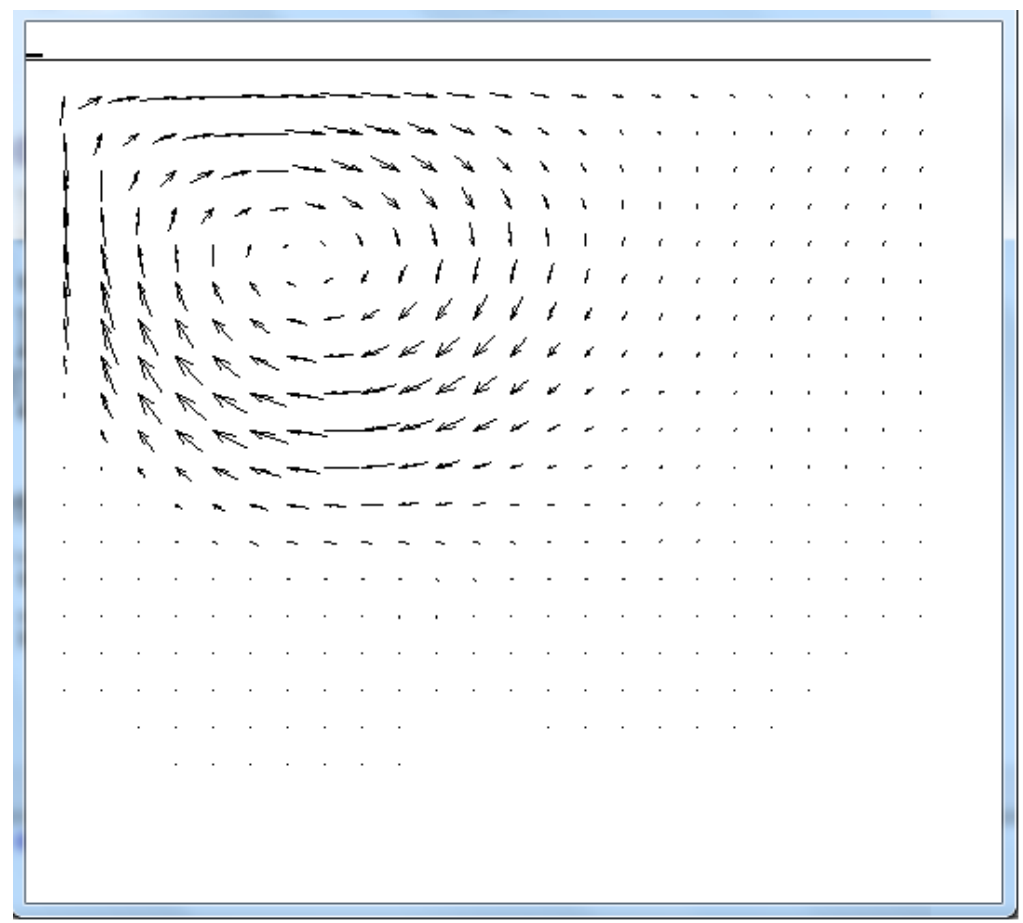

Fig.4. Results of calculating the direction and speed of the wind depending on the variables of the stream function and vortex of velocities. 


\section{Conclusion}

Thus, a software tool developed based on the above mathematical support of the problem under study makes it possible to calculate the following indicators:

- boundaries of areas corresponding to streamlines;

- the direction and speed of the wind, depending on the variables of the stream function and vortex velocities.

- the number of aerosols dropped on the underlying surface;

- the number of aerosols dropped onto the underlying surface at a certain point in time at a given point.

Computational experiments have shown that the change in the concentration of aerosols in the atmosphere depends on the absorption coefficient of particles, which varies depending on meteorological parameters, season, and time of day.

It has been established that the change in the concentration of aerosol emissions in the atmosphere is directly influenced by the direction and speed of the air flow in the atmosphere, the mass of particles, the location, and the number of aerosol generators in the region under consideration. With an increase in the power of aerosol generators, the area of the region grows, where the concentration exceeds the permissible sanitary standard.

The simulation results revealed that elevations - hills or mountain ranges located in an open landscape - play a significant role in changing the wind speed and direction. Above the hills, the wind speed is higher compared to the surrounding flat territory. Since the highpressure area actually expands some distance up the hill, the wind changes direction before reaching it. If the air mass meets a steep hill with an uneven surface, then the wind speed increases sharply, which leads to an increase in the turbulence coefficient. The wind speed increases with an increase in the atmospheric pressure difference, and the air flow speed decreases near the ground due to friction on the roughness of the underlying surface.

The analysis of the performed computational experiments shows that in predicting atmospheric pollution, a special role is played by taking into account the coefficient of interaction with the underlying surface;

The software can be successfully used for research, analysis, and prediction of the process of spreading harmful substances in the atmosphere.

\section{References}

1. A. Ye. Aloyan, Rossiyskaya akademiya nauk Institut vychislitel'noy matematiki Kafedra matematicheskogo modelirovaniya fizicheskikh protsessov MFTI, (2002)

2. CH. I. A., "Matematicheskaya model' optimizatsionnoy zadachi razmeshcheniya pozharoopasnykh ob"yektov s uchetom rel'yefa oblasti razmeshcheniya," Zaporozhskiy natsional'nyy tekhnicheskiy universitet, (2021)

3. A. Kordzadze, Mathematical modelling of dynamical and ecological processes in the system sea-land-atmosphere, NATO Secur. through Sci. Ser. C Environ. Secur., doi: 10.1007/978-1-4020-5877-6_17.pp. 181-193, (2007),

4. M. Sharan and S. G. Gopalakrishnan, Mathematical modeling of diffusion and transport of pollutants in the atmospheric boundary layer, Pure Appl. Geophys., 160, (1-2), pp. 357-394, Jan. 2003, doi: 10.1007/s00024-003-8784-5. (2003).

5. Y. Khan, M. Shekhu, and C. Sulochana, Mathematical model for dispersion and diffusion of chemically reactive pollutants from various sources into a boundary layer with dry deposition, Eng. Comput. (Swansea, Wales), doi: 10.1108/EC-08-2013-0098. 30, (5), pp. 707-727, (2013),

6. Laykhtman David L'vovich, Kniga 'Fizika pogranichnogo sloya atmosfery, Gidrometeorologicheskoye izdatel'stvo, (1970) 
7. Y. Khan, M. Shekhu, and C. Sulochana, Mathematical model for dispersion and diffusion of chemically reactive pollutants from various sources into a boundary layer with dry deposition, Eng. Comput. (Swansea, Wales), doi: 10.1108/EC-08-2013-0098. 30, (5), pp. 707-727, (2013),

8. Laykhtman David L'vovich, Kniga 'Fizika pogranichnogo sloya atmosfery, Gidrometeorologicheskoye izdatel'stvo, (1970)

9. Marchuk G.I., Matematicheskoye modelirovaniye v probleme okruzhayushchey sredy (2011)

10. F. N. Yasinskiy, Mathematical Modeling of the Processes to Ventilations and Heating in Greater Production, Cultural and Atheletic Premiseses, (2021)

11. D. Sharipov, F. Muradov, and D. Akhmedov, Numerical modeling method for shortterm air quality forecast in industrial regions, Appl. Math. E - Notes, 19, pp. 575-584, (2019).

12. Mayyer R.V., "R. V. Mayyer Zadachi, algoritmy, programmy, (2012)

13. Ravshanov N., SHaripov D.K., and Akhmedov D., Modelirovaniye protsessa zagryazneniya okruzhayushchey sredy $\mathrm{s}$ uchetom rel'yefa mestnosti i pogodnoklimaticheskikh faktorov, Informatsionnyye tekhnologii modelirovaniya i (2021)

14. N. Ravshanov, D. K. Sharipov, and F. Muradov, COMPUTATIONAL EXPERIMENT FOR FORECASTING AND MONITORING THE ENVIRONMENTAL CONDITION OF INDUSTRIAL REGIONS, Theor. Appl. Sci., (2016)

15. D. K. Sharipov, Developing of model and web application for forecasting of ecological state of the atmosphere, Theor. Appl. Sci., 40, (08), pp. 58-69, (2016)

16. D. Sharipov, Z. Abdullaev, Z. Tazhiev, and O. Khafizov, Implementation of a mathematical model of a hexacopter control system, (2019)

17. D. Sharipov, S. Aynakulov, and O. Khafizov, Computer Modeling of Aerosol Emissions Spread in the atmosphere, 2019, (2019)

18. Z. Ravshanov, Z. Abdullaev, and O. Khafizov, Atmospheric dispersion modelling of dust emissions from the dried bottom of the Aral Sea, in IOP Conference Series: Materials Science and Engineering, 896, (1), (2020)

19. N. Ravshanov, S. Daliev, Z. Abdullaev, and O. Khafizov, Ground and confined underground waters and their salt content, in IOP Conference Series: Materials Science and Engineering, Aug. 2020, 896, (1), (2020)

20. SHaripov D.K., Muradov F., and Ravshanov Z.N., Matematicheskaya model' i vychislitel'nyy eksperiment dlya monitoringa i prognozirovaniya ekologicheskogo sostoyaniya pogranichnogo sloya atmosfery, (2017)

21. Sharipov, D., Abdukadirov, A., Khasanov, A., Khafizov, O., Mathematical model for optimal siting of the industrial plants, in 2020 International Conference on Information Science and Communications Technologies, ICISCT 2020, 2020, 9351476. (2020).

22. Z. Ravshanov, Z. Abdullaev, and O. Khafizov, Atmospheric dispersion modelling of dust emissions from the dried bottom of the Aral Sea, in IOP Conference Series: Materials Science and Engineering, Aug. 2020, 896, (1), doi: 10.1088/1757899X/896/1/012045. (2020). 